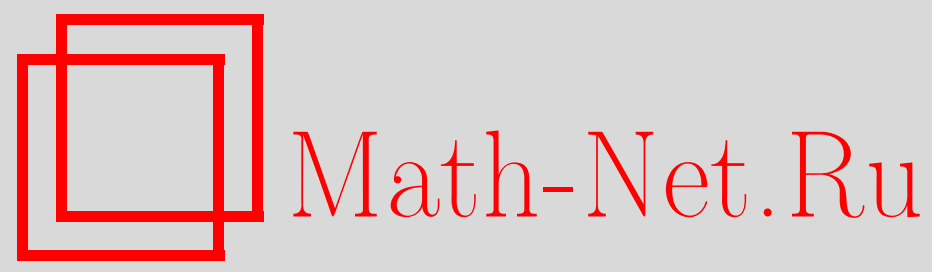

А. Н. Щетинин, О когомологиях эрмитовых симметрических пространств, Матем. заметки, 2003, том 74, выпуск $6,934-943$

DOI: https://doi.org/10.4213/mzm317

Использование Общероссийского математического портала Math-Net.Ru подразумевает, что вы прочитали и согласны с пользовательским соглашением http://www . mathnet.ru/rus/agreement

Параметры загрузки:

IP : 54.81 .137 .203

26 апреля 2023 г., 18:10:05

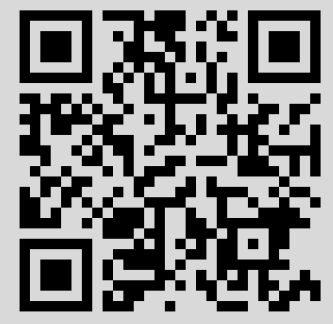




\section{О КОГОМОЛОГИЯХ ЭРМИТОВЫХ СИММЕТРИЧЕСКИХ ПРОСТРАНСТВ}

\section{А. Н. Щетинин}

Пусть $M$ - односвязное компактное эрмитово симметрическое пространство. Выводится формула для значения степени двумерного класса когомологий $M$ на произвольном классе гомологий этого пространства.

Библиография: 8 названий.

1. Введение. При изучении когомологий однородных пространств часто бьвает нужно вычислять значение класса когомологий на классе гомологий. Один из таких результатов получен в [1]. Он дает значение степени элемента $z \in H^{2}(K / L)$ на фундаментальном классе гомологий $[K / L]$, где $K$ - компактная связная полупростая группа Ли, $L$ - ее (связная) подгруппа, являющаяся централизатором некоторого тора в $K$. Такое однородное пространство определяется парой $(R, \Theta)$, где $R$ - система корней группы $K$, $\Theta$ - ее подсистема, порожденная некоторым множеством простых корней. Формула, принадлежащая Борелю и Хирцебруху, имеет вид

$$
\left\langle z^{m},[K / L]\right\rangle=m ! \prod \frac{(z, \gamma)}{(\rho, \gamma)} .
$$

Здесь $2 m$ - размерность $K / L, 2 \rho$ - сумма положительных корней, $(\cdot, \cdot)$ - инвариантное скалярное произведение, и произведение берется по всем положительным корням $\gamma$ из $R \backslash \Theta$.

Мы хотим получить аналогичную формулу для значений степеней двумерного класса когомологий на произвольных классах гомологий пространств вида $K / L$ в случае, когда $K=K_{1} \times \cdots \times K_{r}, L=L_{1} \times \cdots \times L_{r}$, где $K_{j}, j=\overline{1, r},-$ простые неабелевы компактные связные групшы Ли, $L_{j}$ - централизаторы торов в $K_{j}$, являющиеся максимальными связными подгруппами. Как хорошо известно (см. [2]), это условие эквивалентно тому, что

$$
K / L=K_{1} / L_{1} \times \cdots \times K_{r} / L_{r}
$$

- односвязное компактное эрмитово симметрическое пространство. С помощью результата работы [3] поставленная задача сводится к комбинаторной задаче об отыскании числа путей в некотором ориентированном графе. Этот граф явно строится для неприводимого эрмитова симметрического пространства каждого типа. К сожалению, автору не удалось получить доказательство, не использующее классификацию.

Работа выполнена при поддержке Российского фонда фундаментальных исследований, грант № 98-01-00329. 
2. Пути в ориентированных графах. Рассмотрим в $\mathbb{R}^{s}$ решетку $\mathbb{Z}^{s}$ и ориентированный граф $\Gamma^{s}$, вершинами которого являются точки этой решетки, а дуги соединяют точки вида $\left(a_{1}, \ldots, a_{i}-1, \ldots, a_{s}\right)$ и $\left(a_{1}, \ldots, a_{i}, \ldots, a_{s}\right)$. Рассмотрим в графе $\Gamma^{s}$ подграф $\Gamma_{1}^{s}$, состоящий из таких вершин $\left(a_{1}, \ldots, a_{s}\right)$, что $0 \leqslant a_{1}<a_{2}<\cdots<a_{s}$, и соединяющих их дуг.

Теорема 1. Число путей в графе $\Gamma_{1}^{s}$, соединяющих вериину $(0,1, \ldots, s-1) c$ вершиной $\left(a_{1}, \ldots, a_{s}\right)$, при $s=1$ равно $Q\left(a_{1}\right)=1$ и при $s>1$ равно

$$
Q\left(a_{1}, \ldots, a_{s}\right)=D\left(a_{1}, \ldots, a_{s}\right) \frac{\left(a_{1}+\cdots+a_{s}-b\right) !}{a_{1} ! \ldots a_{s} !},
$$

əде $b=s(s-1) / 2, a$

$$
D\left(a_{1}, \ldots, a_{s}\right)=\prod_{1 \leqslant i<j \leqslant s}\left(a_{j}-a_{i}\right)
$$

ДокАЗАТЕЛьСтво. Эта теорема следует из теоремы 3.73 и предложения 3.68 из [4]. Мы приведем все же более короткое доказательство.

Применим индукцию по $a_{1}+\cdots+a_{s}$. Начало индукции очевидно. Рассмотрим вершину $\left(a_{1}, \ldots, a_{s}\right)$. В нее ведут пути в точности из вершин $\left(a_{1}, \ldots, a_{i}-1, \ldots, a_{s}\right)$, если $a_{i-1}<a_{i}-1$ (считаем $\left.a_{0}=-1\right)$. Положим

$$
A=\frac{\left(a_{1}+\cdots+a_{s}-b-1\right) !}{a_{1} ! \ldots a_{s} !} .
$$

По предположению индукции и учитьвая, что $D\left(a_{1}, \ldots, a_{i}-1, \ldots, a_{s}\right)=0$ при $a_{i-1}=$ $a_{i}-1, i>1$, имеем

$$
\begin{aligned}
& Q\left(a_{1}, \ldots, a_{s}\right)=\sum_{a_{i-1}<a_{i}-1} Q\left(a_{1}, \ldots, a_{i}-1, \ldots, a_{s}\right) \\
& \quad=A \sum_{a_{i-1}<a_{i}-1} a_{i} D\left(a_{1}, \ldots, a_{i}-1, \ldots, a_{s}\right)=A \sum_{i=1}^{s} a_{i} D\left(a_{1}, \ldots, a_{i}-1, \ldots, a_{s}\right) .
\end{aligned}
$$

Рассмотрим сумму $\sum_{i=1}^{s} a_{i} D\left(a_{1}, \ldots, a_{i}-1, \ldots, a_{s}\right)$. Как легко видеть, это (неоднородный) кососимметрический многочлен от $a_{1}, \ldots, a_{s}$. Значит,

$$
\sum_{i=1}^{s} a_{i} D\left(a_{1}, \ldots, a_{i}-1, \ldots, a_{s}\right)=D\left(a_{1}, \ldots, a_{s}\right) f\left(a_{1}, \ldots, a_{s}\right)
$$

где $f$ - симметрический многочлен. Имеем $f\left(a_{1}, \ldots, a_{s}\right)=a_{1}+\cdots+a_{s}+c$, и нам надо вычислить константу $c$. Для этого положим $a_{i}=i-1, i=\overline{1, s}$. Подставив это в (2), видим, что левая часть равна нулю, а $D(0,1, \ldots, s-1) \neq 0$. Значит, $f(0,1, \ldots, s-1)=0$, откуда $c=-s(s-1) / 2$, как и требовалось.

Рассмотрим теперь подграф $\Gamma_{2}^{s}$ в $\Gamma^{s}$, состояший из вершин $\left(0, \ldots, 0, a_{1}, \ldots, a_{k}\right), 1 \leqslant$ $a_{1}<\cdots<a_{k}, k=\overline{0, s}$, и соединяюших их дуг. Такие вершины будем обозначать просто $\left(a_{1}, \ldots, a_{k}\right)$. Пометим дуги этого графа, соединяющие вершины с одинаковым числом координат, натуральным числом $\lambda$, а остальные дуги - числом 1 . Мы получим размеченньй граф $\Gamma_{2}^{s}(\lambda)$. При определении числа путей в таком графе будем каждую дугу считать с кратностью, равной числовой отметке. 
ТЕорема 2. Число путей в графе $\Gamma_{2}^{s}(\lambda)$, соединяющих начало координат с вершиной $\left(a_{1}, \ldots, a_{k}\right)$, при $k=1$ равно $Q\left(a_{1}\right)=\lambda^{a_{1}-1}$ и при $k>1$ равно

$$
Q\left(a_{1}, \ldots, a_{k}\right)=\lambda^{a_{1}+\cdots+a_{k}-k} \frac{\left(a_{1}+\cdots+a_{k}\right) !}{a_{1} ! \ldots a_{k} !} \prod_{i<j} \frac{a_{j}-a_{i}}{a_{j}+a_{i}} .
$$

ДокАЗАТЕЛЬСТво. Индукция по $a_{1}+\cdots+a_{k}$. Полагая $Q_{i}=Q\left(a_{1}, \ldots, a_{i}-1, \ldots, a_{k}\right)$, если $a_{i-1}<a_{i}-1$, и $Q_{i}=0$, если $a_{i-1}=a_{i}-1, i=\overline{1, k}, a_{0}=-1$, имеем

$$
\begin{array}{ll}
Q\left(a_{1}, \ldots, a_{k}\right)=\lambda \sum_{i=1}^{k} Q_{i}, & a_{1}>1, \\
Q\left(a_{1}, \ldots, a_{k}\right)=Q\left(a_{2}, \ldots, a_{k}\right)+\lambda \sum_{i=2}^{k} Q_{i}, & a_{1}=1 .
\end{array}
$$

По предположению индукции (считая, что $k>1$ )

$$
Q_{i}=\frac{A a_{i} D\left(a_{1}, \ldots, a_{i}-1, \ldots, a_{k}\right)}{\prod_{r<t, r, t \neq i}\left(a_{r}+a_{t}\right) \prod_{r \neq i}\left(a_{r}+a_{i}-1\right)}, \quad i=\overline{1, k},
$$

где общий множитель

$$
A=\lambda^{a_{1}+\cdots+a_{k}-k-1} \frac{\left(a_{1}+\cdots+a_{k}-1\right) !}{a_{1} ! \ldots a_{k} !},
$$

и $Q\left(a_{2}, \ldots, a_{k}\right)=\lambda Q_{1}$ при $a_{1}=1$.

Рассмотрим дробь $Q_{i} / A+Q_{j} / A$. Она равна дроби, знаменатель которой имеет вид

$$
\prod_{r<t, r, t \neq i, j}\left(a_{r}+a_{t}\right) \prod_{r \neq i, j}\left(a_{r}+a_{i}-1\right) \prod_{r \neq j}\left(a_{r}+a_{j}-1\right),
$$

а числитель, с точностью до знака, есть

$$
\begin{aligned}
& \left(a_{i} \prod_{r \neq i}\left(a_{r}-a_{i}+1\right) \prod_{r \neq i, j}\left(a_{r}-a_{j}\right) \prod_{r \neq i, j}\left(a_{r}+a_{i}\right) \prod_{r \neq i, j}\left(a_{r}+a_{j}-1\right)\right. \\
& -a_{j} \prod_{r \neq j}\left(a_{r}-a_{j}+1\right) \prod_{r \neq i, j}\left(a_{r}-a_{i}\right) \prod_{r \neq i, j}\left(a_{r}+a_{j}\right) \\
& \left.\quad \times \prod_{r \neq i, j}\left(a_{r}+a_{i}-1\right)\right) \prod_{r<t, r, t \neq i, j}\left(a_{r}-a_{t}\right) .
\end{aligned}
$$

Как легко проверить, этот многочлен обращается в 0 при $a_{i}=1-a_{j}$ и потому делится на $a_{i}+a_{j}-1$. Таким образом, после приведения к одному знаменателю суммы (3) или (4) сомножители этого вида сократятся и останется знаменатель $\prod_{r<t}\left(a_{r}+a_{t}\right)$. Так как все дроби $Q_{i} / A$ кососимметричны по $a_{1}, \ldots, a_{s}$, то вся сумма примет вид

$$
Q\left(a_{1}, \ldots, a_{k}\right)=\frac{\lambda A D\left(a_{1}, \ldots, a_{k}\right) f\left(a_{1}, \ldots, a_{k}\right)}{\prod_{i<j}\left(a_{i}+a_{j}\right)},
$$

где $f$ - симметрический многочлен. Сравнивая степени, видим, что $f=a_{1}+\cdots+a_{k}+c$. Положим теперь $a_{i}=i, i=\overline{1, k}$. Тогда все $Q\left(a_{1}, \ldots, a_{i}-1, \ldots, a_{k}\right)=0$, за исключением отвечающего $i=1$, откуда $f(1,2, \ldots, k)=k(k+1) / 2$. Значит, константа $c=0$, и мы получаем требуемое утверждение. 
СледСтвиЕ. Рассмотрим в графе из теоремы 2 подмнохсество, состоящее из вершин с четным числом координат. Дуги, соединяющие вериины с одинаковым числом координат, такие же, как в графе из теоремы 2. И пусть, кроме того, дуги идут от вериин $\left(a_{1}, \ldots, a_{2 k}\right) \kappa$ вершинам $\left(1,2, a_{1}, \ldots, a_{2 k}\right), 0 \leqslant 2 k \leqslant s-2, a$ все числовые отметки равны 1. Тогда число путей, ведущих из начала координат в вериину $\left(a_{1}, \ldots, a_{2 k}\right)$, равно

$$
Q^{\prime}\left(a_{1}, \ldots, a_{2 k}\right)=\frac{\left(a_{1}+\cdots+a_{2 k}-2 k\right) !}{\left(a_{1}-1\right) ! \ldots\left(a_{2 k}-1\right) !} \prod_{i<j} \frac{a_{j}-a_{i}}{a_{j}+a_{i}-2} .
$$

ДокАЗАТЕЛЬСТво вытекает из того, что $Q^{\prime}\left(a_{1}, \ldots, a_{2 k}\right)=Q\left(a_{1}-1, \ldots, a_{2 k}-1\right)$, где $Q\left(a_{1}, \ldots, a_{l}\right)$ - функция из теоремы 2, а $\lambda=1$.

3. Граф однородного пространства. Пусть $K$ - компактная связная полупростая группа Ли, $T$ - ее максимальный тор, $R$ - система корней групшы $K$ относительно тора $T$. Зафиксируем базис $B=\left\{\alpha_{1}, \ldots, \alpha_{n}\right\}$ системы корней $R$, и пусть $R_{+}-$соответствующее множество положительных корней. Простые корни будем нумеровать как в [5]. Обозначим через $\beta$ максимальньй корень и через $2 \rho$ - сумму положительных корней.

Введем инвариантное скалярное произведение $(\cdot, \cdot)$, нормированное условием $(\gamma, \gamma)=2$ для короткого корня $\gamma$. Для корня $\gamma$ через $s_{\gamma}$ обозначим отражение относительно $\gamma$. Пусть $W$ - група Вейля системы $R$. Если $\Theta$ - подсистема в $R$, имеющая в качестве базиса некоторое подмножество из $B$, то положим $\Theta_{+}=\Theta \cap R_{+}$и через $W(\Theta)$ обозначим подгруппу в $W$, порожденную отражениями $s_{\gamma}, \gamma \in \Theta$. Через $\omega_{i}$ обозначим $i$-й фундаментальньй вес.

Для элемента $w \in W$ обозначим через $l(w)$ его длину, т.е. такое минимальное число $q$, что $w$ есть произведение $q$ отражений вида $s_{\alpha}, \alpha \in B$. Группа $W$ допускает представление в виде ориентированного графа, вершины которого соответствуют ее элементам, а два элемента $w$ и $w^{\prime}$ соединяются дугой $w \rightarrow w^{\prime}$, если $l\left(w^{\prime}\right)=l(w)+1, w^{\prime}=s_{\gamma} w$ для некоторого $\gamma \in R_{+}$.

Подсистема $\Theta$ определяет централизатор тора в $K$, которьй мы обозначим через $L$. Рассмотрим множество смежных классов $W / W(\Theta)$. Как показанов [3], в каждом смежном классе содержится единственньй представитель наименьшей длины и это множество представителей

$$
W^{1}(\Theta)=\left\{w \in W \mid w\left(\Theta_{+}\right) \subset R_{+}\right\} .
$$

Отметим, что если $w \in W^{1}(\Theta), \gamma \in R_{+}$и $w^{-1}(\gamma)<0$, то $w^{-1}(\gamma) \notin \Theta$.

Рассмотрим в графе группы $W$ вершины, отвечающие элементам из $W^{1}(\Theta)$, и дуги, их соединящие. Мы получим подграф, которьй назовем графом однородного пространства $K / L$. При этом, если $w, w^{\prime} \in W^{1}(\Theta), w \rightarrow w^{\prime}$ и $w^{\prime}=s_{\gamma} w$, то $w^{\prime}=w s_{w^{-1}(\gamma)}$ и $w^{-1}(\gamma) \notin \Theta$, так как в противном случае элементы $w$ и $w^{\prime}$ принадлежали бы одному смежному классу по $W(\Theta)$.

Пусть $w_{0}-$ (единственньй) элемент максимальной длины в $W, w_{\Theta}$ - (также единственньй) элемент максимальной длины в $W(\Theta), w_{\Theta}^{1}=w_{0} w_{\Theta}$. Предположим, что $\gamma \in R_{+}$. Тогда $w_{\Theta}^{1}(-\gamma) \in R_{+}$в точности, когда $\gamma \in R_{+} \backslash \Theta_{+}$. Ясно, что $w_{\Theta}^{1} \in W_{\Theta}^{1}$ и это единственный элемент максимальной длины в $W_{\Theta}^{1}$.

Приведем теперь необходимые сведения о гомологиях и когомологиях однородного пространства $K / L$. Мы рассматриваем исключительно целочисленные гомологии и 
когомологии. Гомологии $H_{2 l}(K / T)$ пространства $K / T$ образуют свободную абелеву группу с образуюшими $D_{w}, w \in W, l(w)=l$. Естественное отображение $p: K / T \rightarrow$ $K / L$ индуцирует эпиморфизм абелевых групп $p_{*}: H_{*}(K / T) \rightarrow H_{*}(K / L)$ и мономорфизм колец $p^{*}: H^{*}(K / L) \rightarrow H^{*}(K / T)$. Обозначая через $\langle x, y\rangle$ значение класса когомологий $x$ на классе гомологий $y$, имеем

$$
\left\langle p^{*}(z), D_{w}\right\rangle=\left\langle z, p_{*}\left(D_{w}\right)\right\rangle,
$$

где $z \in H^{*}(K / L)$. При этом $p_{*}\left(D_{w}\right)=0$, если $w \notin W^{1}(\Theta)$, а элементы $F_{w}=p_{*}\left(D_{w}\right)$, $w \in W^{1}(\Theta)$, образуют базис свободной абелевой группы $H_{*}(K / L)$ (см. [3]).

Свободную абелеву группу $H^{2}(K / T)$ отождествим с решеткой весов групшы $K$ (которую без потери общности считаем односвязной). Если $z \in H^{2}(K / L)$, то $p^{*}(z)$ можно отождествить с некоторым весом. Мы будем вместо $p^{*}(z)$ писать просто $z$. Положим $z(\gamma)=2(z, \gamma) /(\gamma, \gamma), \gamma \in R$

Вплоть до п. 5 групу $K$ считаем простой. Пусть подсистема $\Theta$ имеет базис $B \backslash\left\{\alpha_{i}\right\}$, и пусть $L$ - соответствуюший централизатор тора. Снабдим дугу $w \rightarrow w^{\prime}$ графа пространства $K / L$ числовой отметкой, равной $\omega_{i}(\gamma)$, где $w^{\prime-1}=s_{\gamma} w^{-1}, \gamma \in R_{+}$. Мы получим размеченный граф $\Gamma(K / L)$ однородного пространства $K / L$.

Предположим, что $L-$ максимальная связная подгруппа. Имеем $\beta=\sum_{j=1}^{n} c_{j} \alpha_{j}$, причем $c_{i}=1$ (см., например, [6]). Если $\gamma \in R_{+} \backslash \Theta_{+}$, то

$$
\gamma=\sum_{j=1}^{n} d_{j} \alpha_{j}, \quad d_{j} \leqslant c_{j}, \quad j=\overline{1, n}
$$

и $d_{i} \neq 0$, откуда $d_{i}=1$ и $\left(\omega_{i}, \gamma\right)=\left(\omega_{i}, \alpha_{i}\right)$. Таким образом, все числовые отметки равны 1 , кроме случая, когда $R$ - система типа $B_{n}$ или $C_{n}$, а $\gamma-$ короткий корень (здесь $\alpha_{i}$ - длинный корень, и соответствующие отметки равны 2).

Пусть $z \in H^{2}(K / L)$ и $w \in W^{1}(\Theta)$. Тогда (см. [3, теорема 3.12])

$$
\left\langle z^{l}, F_{w}\right\rangle=\sum z\left(\gamma_{1}\right) \ldots z\left(\gamma_{l}\right)
$$

где сумма берется по всем цепочкам

$$
e \rightarrow w_{1} \rightarrow \cdots \rightarrow w_{l}=w^{-1}, \quad w_{i}=s_{\gamma_{i}} w_{i-1}, \quad \gamma_{i}>0, \quad i=\overline{1, l} .
$$

Заметим, что ненулевой вклад в сумму вносят лишш те цепочки, у которых все $w_{i}^{-1} \in$ $W^{1}(\Theta)$. При этом, если $\Theta$ имеет базис $B \backslash\left\{\alpha_{i}\right\}$, а $z=\omega_{i}$, то $z\left(\gamma_{i}\right)$ есть числовая отметка в размеченном графре однородного пространства $K / L$ и правая часть (5) равна числу путей, соединяюших вершину е с вершиной $w$.

Пусть $\Theta$ имеет базис $B \backslash\left\{\alpha_{i}\right\}$. Рассмотрим представление комплексной алгебры Ли, определяемой группой $K$, со старшим весом $\Lambda=\omega_{i}$. Как хорошо известно, $w(\Lambda)$ есть вес того же представления для любого $w \in W$. Поставив в соответствие элементу $w \in$ $W^{1}(\Theta)$ вес $w(\Lambda)$, мы получим отображение множества $W^{1}(\Theta)$ в множество всех весов представления со старшим весом $\Lambda$. Это отображение инъективно. В самом деле, если $w_{1}(\Lambda)=w_{2}(\Lambda), w_{1}, w_{2} \in W^{1}(\Theta)$, то $w_{1}=w_{2} w$, где $w(\Lambda)=\Lambda$. Тогда $w \in W(\Theta)($ см. [7, дополнение]) и $w=1$.

Пусть $w \in W^{1}(\Theta), \alpha \in B$ и $(w(\Lambda), \alpha)>0$. Тогда $w^{-1}(\alpha)>0$ и $w^{-1}(\alpha) \notin \Theta$, откуда $l\left(s_{\alpha} w\right)=l(w)+1$ (см. [7]) и $w^{\prime}=s_{\alpha} w \in W^{1}(\Theta)$. При этом $w(\Lambda)-\Lambda(\alpha) \alpha=w^{\prime}(\Lambda)$ есть вес. Обратно, если $\alpha \in B, w, w^{\prime} \in W^{1}(\Theta), w \rightarrow w^{\prime}$ и $w^{\prime}=s_{\alpha} w$, то $(w(\Lambda), \alpha)>0$. 
Рассмотрим теперь граф, вершины которого задаются элементами $w \in W^{1}(\Theta)$, а вершины $w$ и $w^{\prime}$ соединены дугой тогда и только тогда, когда существует такой $\alpha \in B$, что

$$
(w(\Lambda), \alpha)>0 \quad \text { и } \quad w^{\prime}(\Lambda)=w(\Lambda)-\Lambda(\alpha) \alpha .
$$

Введем в нем разметку как в $\Gamma(K / L)$. Назовем этот граф $\Gamma(\Lambda)$ әрафом системы весов, хотя не все веса представления, вообе говоря, соответствуют его вершинам. Отметим также (см. ниже замечание после теоремы 3$)$, что в графе $\Gamma(K / L)$ могут быть дуги, которых нет в графе $\Gamma(\Lambda)$.

4. Основная теорема. Рассмотрим формулу (1). Используя результат упражнения 3 из [8, гл. $8, \S 9]$, а также следствие из предложения 29 из [5, гл. $6, \S 1]$, ее можно переписать в более удобном для вычислений виде

$$
\left\langle z^{m},[K / L]\right\rangle=m ! \frac{f_{1} ! \ldots f_{s} !}{k_{1} ! \ldots k_{n} !} \prod z(\gamma)
$$

где произведение берется по всем $\gamma \in R_{+} \backslash \Theta_{+}, k_{1}, \ldots, k_{n}$ - показатели групшы $K$, $f_{1}, \ldots, f_{s}-$ показатели групшы $L$.

Формула (1) справедлива для любого централизатора тора $L$ в произвольной полупростой групе $K$. Мы хотим получить ее обобщение, вычислив значение степени класса когомологий $z$ на произвольном классе гомологий пространства $K / L$. Для простой группы $K$ это удается сделать в случае, когда $L$ - максимальная связная подгруппа.

ТЕОремА 3. Пусть $K$ - простая компактная связная односвязная группа Ли, $L$ - ее максимальная связная подгруппа, являющаяся иентрализатором тора $u$ $z \in H^{2}(K / L)$. Тогда значение класса когомологий $z^{l}$ на классе гомологий $F_{w}$, $l(w)=l$, дается формулой

$$
\left\langle z^{l}, F_{w}\right\rangle=l ! \prod \frac{(w(z),-\gamma)}{(\rho, \gamma)}
$$

где произведение берется по всем таким $\gamma \in R_{+}$, что $w^{-1}(\gamma)<0$.

ДокАЗАтЕльство. Однородное пространство $K / L$ определяется парой $\left(R, \alpha_{i}\right)$. Полньй список таких пар следующий:

$$
\begin{array}{ccccc}
\left(A_{n}, \alpha_{s}\right), \quad 1 \leqslant s \leqslant n, & \left(B_{n}, \alpha_{1}\right), & \left(C_{n}, \alpha_{n}\right), & \left(D_{n}, \alpha_{1}\right), & \left(D_{n}, \alpha_{n-1}\right), \\
\left(D_{n}, \alpha_{n}\right), & \left(E_{7}, \alpha_{7}\right), & \left(E_{6}, \alpha_{1}\right), & \left(E_{6}, \alpha_{6}\right) .
\end{array}
$$

Мы рассмотрим эти случаи в отдельности, построив для каждого из них граф системы весов. Затем докажем, что число путей в таком графе, соединяюших вершину е с вершиной $w$, определяется правой частью формулы (7). Можно считать, что $z=\Lambda=\omega_{i}$.

1) Пусть пространство отвечает паре $\left(A_{n}, \alpha_{s}\right)$. Имеем

$$
R_{+}=\left\{\varepsilon_{i}-\varepsilon_{j} \mid 1 \leqslant i<j \leqslant n+1\right\}
$$

и $2 \rho=n \varepsilon_{1}+(n-2) \varepsilon_{2}+\cdots-(n-2) \varepsilon_{n}-n \varepsilon_{n+1}$. Старший вес имеет вид $\Lambda=\omega_{s}=\varepsilon_{1}+$ $\cdots+\varepsilon_{s}$. Произвольньй вес можно записать в виде $w(\Lambda)=\varepsilon_{a_{1}+1}+\cdots+\varepsilon_{a_{s}+1}$, где $w \in W^{1}(\Theta), 0 \leqslant a_{1}<a_{2}<\cdots<a_{s} \leqslant n$. Таким образом, установлена биекция между множеством $W^{1}(\Theta)$ и множеством наборов $\left(a_{1}, \ldots, a_{s}\right)$, где $0 \leqslant a_{1}<\cdots<a_{s} \leqslant n$. 
Элементы $w$ и $w^{\prime}$, определенные наборами $\left(a_{1}, \ldots, a_{i}-1, \ldots, a_{s}\right)$ и $\left(a_{1}, \ldots, a_{i}, \ldots, a_{s}\right)$ соответственно, соединяются дугой $w \rightarrow w^{\prime}$.

Мы видим, что граф системы весов можно рассматривать как подграф графа $\Gamma_{1}^{s}$ из теоремы 1 , содержащий в точности вершины, для которых $a_{s} \leqslant n$. Далее, если $w$ отвечает набору $\left(a_{1}, \ldots, a_{s}\right)$, т.е. весу $\varepsilon_{a_{1}+1}+\cdots+\varepsilon_{a_{s}+1}$, то (при $s>1$ )

$$
\prod(\rho, \gamma)=\frac{a_{1} ! \ldots a_{s} !}{D\left(a_{1}, \ldots, a_{s}\right)}, \quad \prod(w(z),-\gamma)=1
$$

где произведение берется по всем $\gamma \in R_{+}, w^{-1}(\gamma)<0$, а число таких корней $\gamma$ есть $l=a_{1}+\cdots+a_{s}-s(s-1) / 2$. Требуемое утверждение вытекает из теоремы 1 .

2) Пара $\left(R, \alpha_{i}\right)$ есть $\left(C_{n}, \alpha_{n}\right)$. Имеем

$$
R_{+}=\left\{\varepsilon_{i} \pm \varepsilon_{j}, \quad 1 \leqslant i<j \leqslant n, \quad 2 \varepsilon_{i}, \quad 1 \leqslant i \leqslant n\right\}
$$

и $\rho=n \varepsilon_{1}+\cdots+2 \varepsilon_{n-1}+\varepsilon_{n}$. Старший вес $\Lambda=\omega_{n}=\varepsilon_{1}+\cdots+\varepsilon_{n}$, остальные веса $w(\Lambda)$ имеют вид $\Lambda-2\left(\varepsilon_{n-a_{1}+1}+\cdots+\varepsilon_{n-a_{k}+1}\right), 1 \leqslant a_{1}<\cdots<a_{k} \leqslant n, 1 \leqslant k \leqslant n$. Граф системы весов можно отождествить с подграфом графа $\Gamma_{2}^{s}(\lambda)$ из теоремы 2 для $\lambda=2, s=n$, состоящим из вершин $\left(a_{1}, \ldots, a_{k}\right)$ с $a_{k} \leqslant n$ и соединяющих их дуг. Если $w$ отвечает такой вершине, то (при $k>1$ )

$$
\prod(\rho, \gamma)=2^{k} a_{1} ! \ldots a_{k} ! \prod_{i<j} \frac{a_{j}+a_{i}}{a_{j}-a_{i}}, \quad \prod(w(z),-\gamma)=2^{a_{1}+\cdots+a_{k}}
$$

где произведение берется по всем $\gamma \in R_{+}, w^{-1}(\gamma)<0$, а число таких корней $\gamma$ равно $l=a_{1}+\cdots+a_{k}$. Требуемое утверждение следует из теоремы 2 .

3) Из случаев $\left(D_{n}, \alpha_{n-1}\right)$ и $\left(D_{n}, \alpha_{n}\right)$ достаточно рассмотреть первьй. Имеем

$$
R_{+}=\left\{\varepsilon_{i} \pm \varepsilon_{j}, \quad 1 \leqslant i<j \leqslant n\right\}
$$

и $\rho=(n-1) \varepsilon_{1}+\cdots+2 \varepsilon_{n-2}+\varepsilon_{n-1}$. Здесь $\Lambda=\omega_{n}=\left(\varepsilon_{1}+\cdots+\varepsilon_{n-1}+\varepsilon_{n}\right) / 2$, остальные веса имеют вид $\Lambda-\left(\varepsilon_{n-a_{1}+1}+\cdots+\varepsilon_{n-a_{2 k}+1}\right), 1 \leqslant a_{1}<\cdots<a_{2 k} \leqslant n, 0 \leqslant 2 k \leqslant n$. Граф системы весов рассматриваем как подграф графа из следствия из теоремы 2 для $s=n$, образованньй всеми вершинами $\left(a_{1}, \ldots, a_{2 k}\right)$ с $a_{2 k} \leqslant n$ и соединяюшими их дугами. Если $w$ отвечает такой вершине, то

$$
\prod(\rho, \gamma)=\left(a_{1}-1\right) ! \ldots\left(a_{2 k}-1\right) ! \prod_{i<j} \frac{a_{j}+a_{i}-2}{a_{j}-a_{i}}, \quad \prod(w(z),-\gamma)=1
$$

произведение берется по всем $\gamma \in R_{+}, w^{-1}(\gamma)<0$ и число таких $\gamma$ равно $l=a_{1}+\cdots+$ $a_{2 k}-2 k$. Теперь применим следствие из теоремы 2 .

4) Случаи $\left(B_{n}, \alpha_{1}\right)$ и $\left(D_{n}, \alpha_{1}\right)$ проверяются элементарно.

$5)$ Рассмотрим случай $\left(E_{7}, \alpha_{7}\right)$. Здесь $R_{+}$состоит из корней вида

$$
\begin{gathered}
\pm \varepsilon_{i}+\varepsilon_{j}, \quad 1 \leqslant i<j \leqslant 6, \quad \varepsilon_{8}-\varepsilon_{7}, \\
\frac{1}{2}\left(\varepsilon_{8}-\varepsilon_{7}+\sum_{i=1}^{6}(-1)^{\nu(i)} \varepsilon_{i}\right) \quad \text { с нечетной суммой } \sum_{i=1}^{6} \nu(i),
\end{gathered}
$$


и $2 \rho=2 \varepsilon_{2}+4 \varepsilon_{3}+6 \varepsilon_{4}+8 \varepsilon_{5}+10 \varepsilon_{6}-17 \varepsilon_{7}+17 \varepsilon_{8}$. Положим

$$
\varepsilon=\frac{1}{2}\left(\varepsilon_{8}-\varepsilon_{7}\right), \quad \sigma=\frac{1}{2}\left(\varepsilon_{1}+\varepsilon_{2}+\varepsilon_{3}+\varepsilon_{4}+\varepsilon_{5}+\varepsilon_{6}\right) .
$$

Старший вес $\Lambda=\omega_{7}=\varepsilon_{6}+\varepsilon$. Граф системы весов можно рассматривать как подграф в $\Gamma^{3}$. Веса соответствуют вершинам графа следуюшим образом. Вес $\varepsilon_{a}+\varepsilon$ отвечает вершине $(6-a, 0,0), 1 \leqslant a \leqslant 6$, вес $-\varepsilon_{b}+\varepsilon-$ вершине $(5, b-1,0), 2 \leqslant b \leqslant 6$, вес $-\varepsilon_{1}+\varepsilon$ - вершине $(4,1,0)$, вес $\sigma$ - вершине $(6,0,0)$, вес $\sigma-\left(\varepsilon_{a}+\varepsilon_{b}\right)$ - вершине $(a+5, b-1,0)$, $1 \leqslant a<b \leqslant 6$ (кроме веса $\sigma-\left(\varepsilon_{5}+\varepsilon_{6}\right)$, отвечающего вершине $\left.(9,6,0)\right)$. Наконец, если вес $\Lambda^{\prime}$ соответствует вершине $(i, j, 0)$, то вес $-\Lambda^{\prime}-$ вершине $(17-i, 9-j, 1)$.

Выпишем по построенному графу производящую функцию числа путей

$$
\begin{aligned}
S(x, y, z)= & +x+x^{2}+x^{3}+x^{4}(1+y)+x^{5}\left(1+2 y+2 y^{2}+2 y^{3}+2 y^{4}+2 y^{5}\right) \\
& +x^{6}\left(1+3 y+5 y^{2}+7 y^{3}+9 y^{4}+11 y^{5}\right)+x^{7}\left(5 y^{2}+12 y^{3}+21 y^{4}+32 y^{5}\right) \\
& +x^{8}\left(12 y^{3}+33 y^{4}+12 y^{3} z+65 y^{5}+45 y^{4} z+110 y^{5} z\right) \\
& +x^{9}\left(33 y^{4}+98 y^{5}+78 y^{4} z+98 y^{6}+286 y^{5} z+384 y^{6} z\right) \\
& +x^{10}\left(78 y^{4} z+364 y^{5} z+748 z\left(y^{6}+y^{7}\right)\right) \\
& +x^{11} z\left(78 y^{4}+442 y^{5}+1190 y^{6}+1938\left(y^{7}+y^{8}+y^{9}\right)\right) \\
& +x^{12} z\left(78 y^{4}+520 y^{5}+1710 y^{6}+3648 y^{7}\right)+5586 y^{8} z\left(x^{12}+x^{13}\right) \\
& +7524 x^{12} y^{9} z+13110 y^{9} z\left(x^{13}+x^{14}+x^{15}+x^{16}+x^{17}\right) .
\end{aligned}
$$

Для вершины $(i, j, k)$, отвечающей весу $\sigma-\left(\varepsilon_{a}+\varepsilon_{b}\right)$, правая часть $(7)$ дает

$$
D(a, b, 2 a+2 b-2) \frac{(a+b-3) !}{a ! b !}, \quad 1 \leqslant a<b \leqslant 6,
$$

а для вершины, отвечаюшей весу $-\sigma+\left(\varepsilon_{a}+\varepsilon_{b}\right),-$

$$
\begin{gathered}
\frac{k(a) D(a, b, c)(a+4) !(b+4) !(c+4) !(c-7) !}{(a-1) !(b-1) !(c-1) !(17-a)(17-b)(17-c)(6-a) !(6-b) ! 10 ! 7 !} \\
c=19-a-b, \quad 1 \leqslant a<b \leqslant 6,
\end{gathered}
$$

где $k(a)=2$, если $a=1$, и $k(a)=1$ в противном случае. Несложная, хотя длинная проверка показьвает, что мы получаем в точности коэффициент при $x^{i} y^{j} z^{k}$ в $S(x, y, z)$. Для остальных вершин явные формулы мы не вьписьваем, но проверка также не представляет трудностей.

6) Из оставшихся достаточно рассмотреть случай $\left(E_{6}, \alpha_{6}\right)$. Положительные корни имеют вид

$$
\begin{gathered}
\pm \varepsilon_{i}+\varepsilon_{j}, \quad 1 \leqslant i<j \leqslant 5 \\
\frac{1}{2}\left(\varepsilon_{8}-\varepsilon_{7}-\varepsilon_{6}+\sum_{i=1}^{5}(-1)^{\nu(i)} \varepsilon_{i}\right) \quad \text { с четной суммой } \sum_{i=1}^{5} \nu(i),
\end{gathered}
$$

и $\rho=\varepsilon_{2}+2 \varepsilon_{3}+3 \varepsilon_{4}+4 \varepsilon_{5}+4\left(\varepsilon_{8}-\varepsilon_{7}-\varepsilon_{6}\right)$. Положим

$$
\varepsilon=\frac{1}{6}\left(-\varepsilon_{6}-\varepsilon_{7}+\varepsilon_{8}\right), \quad \sigma=\frac{1}{2}\left(\varepsilon_{1}+\varepsilon_{2}+\varepsilon_{3}+\varepsilon_{4}+\varepsilon_{5}\right) .
$$


Старший вес $\Lambda=\omega_{6}=\varepsilon_{5}+2 \varepsilon$. Граф системы весов можно рассматривать как подграф в $\Gamma^{2}$. Вес $\varepsilon_{a}+2 \varepsilon$ отвечает вершине $(5-a, 0), 1 \leqslant a \leqslant 5$, вес $-\varepsilon_{a}+2 \varepsilon-$ вершине $(4, a-1), 2 \leqslant a \leqslant 5$, вес $-\varepsilon_{1}+2 \varepsilon-$ вершине $(3,1)$, вес $\sigma-\varepsilon-$ вершине $(5,0)$, вес $\sigma-\varepsilon-\left(\varepsilon_{a}+\varepsilon_{b}\right)-$ вершине $(a+4, b-1), 1 \leqslant a<b \leqslant 5,(a, b) \neq(4,5)$, вес $\sigma-\varepsilon-\left(\varepsilon_{4}+\varepsilon_{5}\right)$ - вершине $(7,5)$, вес $-\sigma-\varepsilon+\varepsilon_{b}-$ вершине $(8,8-b), 1 \leqslant b \leqslant 5$, вес $-4 \varepsilon-$ вершине $(8,8)$.

Производящая функция числа путей есть

$$
\begin{aligned}
S(x, y)= & +x+x^{2}+x^{3}(1+y)+x^{4}\left(1+2 y+2 y^{2}+2 y^{3}+2 y^{4}\right) \\
& +x^{5}\left(1+3 y+5 y^{2}+7 y^{3}+9 y^{4}\right)+x^{6}\left(5 y^{2}+12 y^{3}+21 y^{4}\right) \\
& +x^{7}\left(12 y^{3}+33 y^{4}+33 y^{5}\right)+x^{8}\left(12 y^{3}+45 y^{4}+78\left(y^{5}+y^{6}+y^{7}+y^{8}\right)\right) .
\end{aligned}
$$

Для вершины $(i, j)$, отвечающей весу $\sigma-\varepsilon-\left(\varepsilon_{a}+\varepsilon_{b}\right)$, правая часть формулы (7) дает

$$
D(a, b, 2 a+2 b-2) \frac{(a+b-3) !}{a ! b !}, \quad 1 \leqslant a<b \leqslant 5,
$$

а для вершины, отвечающей весу $-\sigma-\varepsilon+\varepsilon_{b},-$

$$
D(b, 6,13-b) \frac{(b+3) !(16-b) !}{11.7 ! 5 !(b-1) !(12-b) !}, \quad 2 \leqslant b \leqslant 5,
$$

т.е. получаем коэффициент при $x^{i} y^{j}$ в $S(x, y)$, и это нетрудно проверить для остальных вершин.

Для завершения доказательства теоремы остается установить, что графф системы весов совпадает с графом однородного пространства. Заметим, что для вершины $w_{\Theta}^{1}$ число путей для графа однородного пространства, которое дает известная формула (6), совпадает с числом путей в графе системы весов. Кроме того, для каждой вершины $w$ сушествует путь, соединяющий ее с $w_{\Theta}^{1}$. Поэтому, если бы в графе однородного пространства были дуги, которых нет в графе системы весов, то число путей, ведущих в $w_{\Theta}^{1}$, в нем было бы больше. Так как это не так, в действительности эти графы совпадают, и теорема доказана.

ЗАмЕЧАнИЕ. Если система $R$ состоит из корней одной длины, а $L$ - централизатор тора, являющийся максимальной связной подгруппой, то число элементов в $W^{1}(\Theta)$ равно размерности представления, определенного старшим весом $\Lambda=\omega_{i}$ (см. [8]), и вершшнами графа исчерпываются все веса представления.

Если $L$ - немаксимальная подгруппа, то графы $\Gamma(K / L)$ и $\Gamma(\Lambda)$ могут не совпадать. Например, это так для пространства, определенного парой $\left(C_{3}, \alpha_{2}\right)$. Здесь $\Lambda=\varepsilon_{1}+\varepsilon_{2}$ и вершины $w_{1}$ и $w_{2}$, где $w_{1}(\Lambda)=\varepsilon_{2}-\varepsilon_{3}, w_{2}(\Lambda)=-\varepsilon_{1}+\varepsilon_{2}$, не соединены в $\Gamma(\Lambda)$, но соединены в $\Gamma(K / L)$.

5. Случай приводимого пространства. Пусть $M-$ произвольное односвязное компактное эрмитово симметрическое пространство. Тогда

$$
M=K_{1} / L_{1} \times \cdots \times K_{r} / L_{r},
$$

где $K_{i} / L_{i}, i=\overline{1, r},-$ однородные пространства рассмотренного вьше типа. Имеем $R=$ $\bigoplus_{i=1}^{r} R_{i}$, где $R_{i}$ - системы корней груп $K_{i}$ соответственно. Обозначим через $W_{i}$ групу Вейля системы $R_{i}$ и через $2 \rho_{i}$ сумму положительных корней из $R_{i}$. Если пространство $M=K / L$ задается подсистемой корней $\Theta$, то положим $\Theta \cap R_{i}=\Theta_{i}, i=\overline{1, r}$. Имеем

$$
W=W_{1} \times \cdots \times W_{r}, \quad W(\Theta)=W\left(\Theta_{1}\right) \times \cdots \times W\left(\Theta_{r}\right),
$$

и нетрудно проверить, что элемент $w \in W^{1}(\Theta)$ однозначно представляется в виде $w=$ $w_{1} \ldots w_{r}$, где $w_{i} \in W_{i} \cap W^{1}\left(\Theta_{i}\right), i=\overline{1, r}$. 
Tеорема 4. Пусть $z \in H^{2}(M)$. Значение класса когомологий $z^{l}$ на классе гомологий $F_{w}, l(w)=l$, дается формулой

$$
\left\langle z^{l}, F_{w}\right\rangle=l ! \prod \frac{(w(z),-\gamma)}{(\rho, \gamma)},
$$

где произведение берется по всем таким $\gamma \in R_{+}$, что $w^{-1}(\gamma)<0$.

ДокАЗАТЕльСтво. Кольца $H^{*}\left(K_{i} / L_{i}\right), i=\overline{1, r}$, можно рассматривать как подкольца в $H^{*}(K / L)$. Имеем $z=z_{1}+\cdots+z_{r}, z_{i} \in H^{2}\left(K_{i} / L_{i}\right), i=\overline{1, r}$. Пусть $w=w_{1} \ldots w_{r}$, $w_{i} \in W_{i} \cap W^{1}\left(\Theta_{i}\right), l\left(w_{i}\right)=l_{i}, i=\overline{1, r}$. Тогда

$$
\left\langle z^{l}, F_{w}\right\rangle=\frac{l !}{l_{1} ! \ldots l_{r} !} \prod_{i=1}^{r}\left\langle z_{i}, F_{w_{i}}\right\rangle .
$$

Так как

$$
\left\langle z_{i}, F_{w_{i}}\right\rangle=l_{i} ! \prod \frac{\left(w_{i}\left(z_{i}\right),-\gamma_{i}\right)}{\left(\rho_{i}, \gamma_{i}\right)}, \quad i=\overline{1, r},
$$

где произведение берется по всем таким $\gamma_{i} \in\left(R_{i}\right)_{+}$, что $w_{i}^{-1}\left(\gamma_{i}\right)<0$, а $\rho=\rho_{1}+\cdots+\rho_{r}$, получаем требуемое.

\section{СПИСОК ЦИТИРОВАННОЙ ЛИТЕРАТУРЫ}

[1] Borel A., Hirzebruch F. Characteristic classes and homogeneous spaces. II // Amer. J. Math. 1959. V. 81. P. 315-382.

[2] Хелгасон С. Дифференциальная геометрия и симметрические пространства. М.: Мир, 1964.

[3] Бернштейн И. Н., Гельфанд И. М., Гельфанд С. И. Клетки Шуберта и когомологии пространств $G / P / /$ УМН. 1973. Т. 28. № 3. С. 3-26.

[4] Айгнер М. Комбинаторная теория. М.: Мир, 1982.

[5] Бурбаки Н. Группы и алгебры Ли. М.: Мир, 1972.

[6] Гото М., Гроссханс Ф. Полупростые алгебры Ли. М.: Мир, 1981.

[7] Стейнберг Р. Лекции о группах Шевалле. М.: Мир, 1975.

[8] Бурбаки Н. Группы и алгебры Ли. М.: Мир, 1978.

Московский государственный технический университет

Поступило

им. Н. Э. Баумана

20.12 .2000 